\title{
Estimación de la huella hídrica en la producción agrícola de lima Tahití en la Cuenca La Angula, Santander, Colombia
}

\author{
Estimation of water footprints in agricultural production from Tahiti lime in La \\ Angula Basin, Santander, Colombia
}

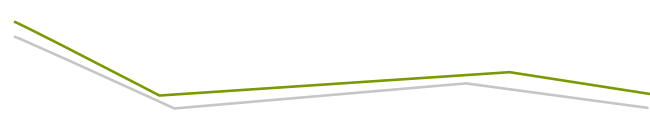

Carlos Fernando Arenas-Jiménez* ${ }^{\star}$, Sandra Natalia Correa-Torres**, Sergio Manuel Pineda-Vargas***

\begin{abstract}
Arenas-Jiménez, C. F., Correa-Torres, S. N., \& Pineda-Vargas, S. M. (2020). Estimación de la huella hídrica en la producción agrícola de lima Tahití en la Cuenca La Angula, Santander, Colombia. Investigación y Ciencia de la Universidad Autónoma de Aguascalientes, 28(79), 52-61.
\end{abstract}

\section{RESUMEN}

Este estudio cuantifica la huella hídrica $(\mathrm{HH})$ azul, verde y gris del cultivo de lima Tahití en la zona alta de la Cuenca de la Quebrada La Angula, Santander, Colombia. Así mismo se analiza la sostenibilidad en el uso del recurso hídrico utilizado en estas actividades. Para esto se utilizó la metodología estándar de cálculo de HH para actividades agrícolas, en donde se determinó el volumen de agua azul y verde adicionada en la producción de lima Tahití y la cantidad del recurso para la depuración de contaminantes en la gris. Para los años estudiados (húmedo y seco) el consumo total para la produc-

Palabras clave: huella hídrica; gestión del recurso hídrico; actividades agrícolas; Cuenca de La Quebrada la Angula.

Keywords: water footprint; water resources management; agricultural activities; La Angula Basin.

Recibido: 11 de febrero de 2019 Aceptado: 22 de noviembre de 2019

* Facultad de Ingeniería Civil, Universidad Pontificia Bolivariana Seccional Montería. Cra. $6 \mathbf{N}^{\circ} 97^{\circ}-99$, C. P. 230002 , Montería, Colombia. Correo electrónico: carlos.arenas@upb.edu.co. DOI: http://orcid.org/0000-00029572-1868

** Facultad de Ingeniería Ambiental, Universidad Pontificia Bolivariana Seccional Bucaramanga. Km 7 vía Floridablanca- Piedecuesta, C. P. 681017, Colombia. Correo electrónico: sandra.correa@upb.edu.co. DOI: http://orcid.org/0000-0002-9572-1868

*** Facultad de Ingeniería Civil, Universidad Pontificia Bolivariana Secciona Bucaramanga. $\mathrm{Km} 7$ vía Floridablanca- Piedecuesta, C. P. 681017 , Colombia. Correo electrónico: sergio.pineda@upb.edu.co. DOI: http://orcid.org/0000-0001-6333-9878

Autor para correspondencia ción anual del fruto estudiado fue $1.74 \times 106 \mathrm{~m}^{3} \mathrm{con}$ una huella virtual de $452,3 \mathrm{~m}^{3} / \mathrm{t}$ para el año húmedo, mientras que para el año seco las $\mathrm{HH}$ total y virtual fueron de $1.25 \times 107 \mathrm{~m}^{3}$ y $486.2 \mathrm{~m}^{3} / \mathrm{t}$, respectivamente.

ABSTRACT

This study quantifies the blue, green and gray water footprint (WF) of the Tahiti lime crop in the upper area of the La Angula ravine basin, Santander, Colombia. Likewise, sustainability in the use of the water resource used in these activities is analyzed. For this, the standard methodology of WF calculation was used for agricultural activities, where the volume of blue and green water added in the production of Tahiti lime and the amount of the resource for the purification of contaminants in the gray was determined. For the years studied (wet and dry), total consumption for annual production of Tahiti lime was $1.74 \times 106 \mathrm{~m}^{3}$ with a virtual footprint of $452.3 \mathrm{~m}^{3} / \mathrm{t}$ for the wet year, while for the dry year, total and virtual were $1.25 \times 107$ $\mathrm{m}^{3}$ and $486.2 \mathrm{~m}^{3} / \mathrm{t}$, respectively.

\section{INTRODUCCIÓN}

Hay mucho por hacer en el tema del análisis y la gestión del agua, aunque se han logrado importantes avances mediante la definición de indicadores de requerimientos hídricos como el agua virtual (AV) y la huella hídrica (HH) (Velázquez, 2008; Zhang, Huang, Yu, \& Yang, 2017). La idea de considerar el uso del agua a lo largo de las cadenas de suministro 
se ha ganado el interés después de que Hoekstra introdujera el concepto de huella de agua (Hoekstra, 2003). La HH puede ser considerada como un indicador integral del aprovechamiento de las fuentes de agua dulce, al lado de la medida tradicional de agua extraída de la fuente.

Para el año 2006 a nivel mundial la $\mathrm{HH}$ fue de $7,450 \mathrm{Gm}^{3} / a n ̃ o$, donde en la India se calculó un valor de $987 \mathrm{Gm}^{3} / a n ̃ o$, mientras en Estados Unidos de 2,480 $\mathrm{m}^{3} /$ año por persona. En Europa se estimó con valores de 2,300 a $2,400 \mathrm{~m}^{3} /$ año por persona, siendo más alta en países del sur como Grecia, Italia y España. En países como China se calculó para

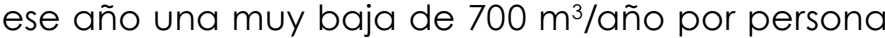
(Hoekstra \& Chapagain, 2006).

En Colombia la aplicación de la metodología para el cálculo de la HH inició con el proyecto SuizAgua, desarrollado por la Agencia para el Desarrollo y la Cooperación Suiza (Cosude) en 2009, el cual se enfocó en estimar los consumos de agua por las actividades agrícolas a nivel nacional (Arévalo Uribe \& Sabogal Mogollón, 2012). En otro estudio, Builes Cedula (2013) determina la HH de la Cuenca del río Porce. Estudios más actuales como el del IDEAM (2015) para Colombia, desarrollaron un análisis de HH azul y verde, junto a un análisis económico para evaluar el comportamiento con relación a las áreas de siembra para 12 cultivos de Colombia en 2012, entre los cultivos estaban arroz, banano, cacao, caña, café, frijol, maíz, plátano, palma de aceite, plátano, soya y yuca (Campuzano, Ochoa, Guzmán, \& Rodríguez, 2015).

Por el incremento del cultivo de limón Tahití en Colombia sería de interés calcular la HH respectiva, ya que en el territorio de dicho país existen cerca de 90,000 ha sembradas con cítricos, donde el limón Tahití ocupa 20\% de los terrenos colombianos (DANE, 2015). En 2014, Colombia produjo cerca de 360,836 † de dos clases de limón, Tahití y Castilla (DANE, 2015). Entre los departamentos del país más productores de limón Tahití en 2014 están Nariño con $70 \%$ de la producción, así como Tolima, Cauca y Santander. En 2013 el departamento de Santander produjo 21,740 t de limón Tahití del total de 35,495 t, seguido de otros como Antioquia, Tolima y Risaralda, información presentada por el Ministerio de Agricultura y Desarrollo Rural.

El caso de la Cuenca Quebrada La Angula tiene una importancia de abastecimiento de alimentos a un casco urbano aproximado de 1,200,000 habitantes en Bucaramanga, Santander. Los cultivos de piña y de lima Tahití son la fuente económica de la población presente en esta Cuenca La Angula, pero los reemplazos de coberturas naturales por actividades agropecuarias están afectando la disponibilidad y calidad del recurso hídrico, además de que existe la problemática del cambio climático que está afectando los cultivos por los extensos periodos de lluvia y sequía. En 2015 el fenómeno El Niño afectó los cultivos de cítricos y redujo el volumen de producción, así como la calidad de la fruta, causada por déficit hídrico y altas temperaturas (IDEAM, 2015).

De acuerdo con lo anterior, se plantea la pregunta de investigación: ¿̇Es sostenible la $\mathrm{HH}$ del cultivo de limón Tahití presente en la zona alta de la Quebrada La Angula del municipio de Lebrija en relación con la disponibilidad del recurso hídrico y sostenibilidad del mismo teniendo en cuenta épocas de lluvia y sequía?

El presente artículo tuvo como objetivo estimar la HH que se genera a partir de la producción agrícola del cultivo de lima Tahití de la zona alta de la Cuenca La Angula, Santander, así como determinar la sostenibilidad de la huella respecto a la oferta hídrica de la zona. El estudio se enfoca en dos años específicos: 2007 y 2015, donde se analizó el cambio del consumo de agua de acuerdo con el régimen de precipitaciones de la zona La Angula. La información recolectada para el cálculo de la HH fue obtenida de las entidades ambientales como Plan de Manejo y Ordenamiento de una Cuenca (POMCA) del Lebrija Alto y la Corporación Autónoma para la Defensa de la Meseta de Bucaramanga Santander, Colombia (CDMB). De acuerdo con la información disponible, se decidió centrar el estudio en dos años con cambios climatológicos extremos, en donde 2007 fue muy húmedo y 2015 muy seco. 


\section{MATERIALES Y MÉTODOS}

\section{Etapa 1: Sistema de información geográfica y procesamiento de información}

La implementación del sistema de información geográfico permitió la recolección y estimación de parámetros climatológicos y de suelo necesarios para el cálculo de la $\mathrm{HH}$ en el lugar de estudio (latitud: 7.005, longitud: -73.2239). La información climatológica se recolectó de estaciones por medio del Instituto de Hidrología, Meteorología y Estudios Ambientales de Colombia (IDEAM), donde se extrajo la información de temperatura, humedad relativa, evaporación y caudales de la Quebrada La Angula.

Los datos del uso de suelo y su cobertura se basaron en los documentos del Plan de Manejo y Ordenamiento de una Cuenca (POMCA) del Lebrija Alto, ubicada en Santander, Colombia y en informes reportados por la Corporación Autónoma para la Defensa de la Meseta de Bucaramanga Santander, Colombia (CDMB) durante los últimos años (CDMB, 2004) (CORPOICA-CDMB, 1997). Estos mapas se procesaron en ArcGis, en formato ráster, superponiendo uno sobre otro para obtener un mapa con todas las características de suelos y coberturas diferenciadas según las diferentes unidades taxonó- micas de la zona delimitada. Las coberturas fueron actualizadas mediante fotografías aéreas tomadas por dron y procesadas digitalmente.

Determinación de caudales. La información de caudales de la Cuenca de la Quebrada La Angula para estimar la oferta de agua azul mensual fue proporcionada por la CDMB. Esta información corresponde a datos tomados en el periodo comprendido desde 1994 hasta 2017. Los datos de caudales disponibles para la zona de estudio se procesaron a partir de la metodología llamada aproximación hidrológica de "gran visión" para estimar los caudales medios mensuales (Salinas-Rodríguez, 2011).

Los caudales ecológicos se calcularon mediante el método de Aproximación hidrológica "detallada", plasmada en la Guía rápida para la determinación de caudales ecológicos (Salinas-Rodríguez, 2011) por medio de la selección de un objetivo ambiental de acuerdo con el estado de conservación que se quiere mantener en la fuente hídrica, dependiendo de factores tales como la presencia de especias amenazadas, zonas de protección o conflictos por el uso del agua. Asimismo, esto depende de si la corriente es perenne o temporal, como se muestra en la tabla 1.

Tabla 1

Valores de referencia para a signar un volumen de caudal ecológico conforme a los objetivos ambientales

\begin{tabular}{cccc}
\hline OBJETIVO & ESTADO DE & \multicolumn{2}{c}{ CAUDAL ECOLÓGICO (\% EMA) } \\
\cline { 3 - 4 } AMBIENTAL & CONSERVACIÓN & $\begin{array}{c}\text { CORRIENTES } \\
\text { PERENNES }\end{array}$ & $\begin{array}{c}\text { CORRIENTES } \\
\text { TEMPORALES }\end{array}$ \\
\hline A & Muy bueno & $\geq 40$ & $\geq 20$ \\
B & Bueno 2 & $5-391$ & $5-19$ \\
C & Moderado 1 & $5-241$ & $0-14$ \\
D & Deficiente 5 & -14 & $5-9$ \\
\hline
\end{tabular}

Nota: Tomada de Salinas-Rodríguez (2011). 


\section{Etapa 2: Cálculo de HH}

La estimación de la HH agrícola conformada por el cálculo de tres específicas, la azul, la verde y la gris, se hizo siguiendo la metodología de Hoekstra et al. (2011).

Cálculo de la $\mathrm{HH}$ verde. Por medio del software Cropwat 8.0 se calculan los requerimientos de agua verde (CWUverde) para un cultivo como la precipitación efectiva (FAO, 2006). Esta representa la fracción de lluvia que realmente es utilizada por las plantas desde la época de siembra hasta el momento de cosecha. La precipitación efectiva se presenta en $\mathrm{mm}$, por lo que el resultado que da el modelo se debe multiplicar por 10 para convertirlo en $\mathrm{m}^{3} / \mathrm{ha}$ o consumo de agua del cultivo.

A partir de estos requerimientos se calcula la $\mathrm{HH}$ $\left(\mathrm{m}^{3} / \mathrm{mes}\right)$ y el agua virtual $\left(\mathrm{m}^{3} / \mathrm{t}\right)$. El modelo que maneja el Cropwat necesita de tres tipos de variables de entrada que corresponden a clima, cultivos y suelos.

Cálculo de la HH azul. Para obtener los requerimientos de agua azul del cultivo (CWUazul) se calculó la diferencia entre el requerimiento del cultivo y la precipitación efectiva. Ambas variables fueron estimadas por el Cropwat. Si el resultado de la diferencia es positivo, ese valor hace referencia al requerimiento de agua azul, mientras que si la resta es igual o menor a cero, no se presenta $\mathrm{HH}$ azul para el periodo y cultivo calculados. Al resultado se le hizo la misma conversión que para los requerimientos de agua verde.

Cálculo de la HH gris. Para calcular el contenido de agua contaminada, se utiliza la siguiente fórmula:

$$
\text { HHcont }=\frac{(a x A R) /(\text { cmax }- \text { cnat })}{\text { Rendimiento }}
$$

donde axAR es el producto de la tasa de aplicación de agroquímicos (AR) por hectárea por fracción de lixiviados (a) dividido entre la máxima concentración aceptable (cmax) menos la concentración natural de sustancias químicas (cnat), todo esto dividido en el rendimiento del cultivo (Hoekstra et al., 2011).

\section{Etapa3: Sostenibilidad de la $\mathrm{HH}$}

Sostenibilidad de la HH verde. Se calcula dividiendo las sumatorias de $H H$ verde mensual, entre la Disponibilidad de Agua verde mensual (Hoekstra et al., 2011) para obtener la Escasez de agua verde mensual (EAVerde) así:

$$
\text { EAVerde }=\frac{\sum H H \text { verde mensual }}{D A \text { verde mensual }}
$$

La disponibilidad de agua verde mensual DAverde mensual se calcula mediante la diferencia entre la evapotranspiración propia de la cuenca ETx estimada por el modelo de Penman-Montheith del módulo de clima del software CROPWAT 8.0, la evapotranspiración de la vegetación natural y de las zonas no productivas, restando los porcentajes de las áreas de estas dos últimas zonas respecto al área total de estudio, es decir, se tuvieron en cuenta solo las áreas cultivadas así:

DAverde mensual

$$
\begin{aligned}
& =E T x, t(\text { total mensual })-E T x, t(\text { nat mensual }) \\
& -E T x, t(\text { improd.mensual })
\end{aligned}
$$

El modelo de Penman-Montheith requiere información para un periodo de tiempo que suele ser anual, aunque puede cambiar dependiendo del tiempo de vida del cultivo a analizar. Los datos de entrada del modelo son temperatura máxima y mínima, humedad relativa, viento e insolación. Si el EAVerde>l, el uso de agua verde no es sostenible pues los requerimientos de los cultivos son mayores a la Disponibilidad de Agua verde. Si el EAVerde<l, la verde es sostenible.

Sostenibilidad de la HH azul. Se debe comparar con la Oferta natural de agua azul mensual (AO natural azul mensual), donde a la Oferta natural mensual de la cuenca (recurso hídrico superficial y subterráneo que sirve como recarga natural) se le resta el Caudal ecológico necesario para el funcionamiento normal y natural del ecosistema fluvial (Hoekstra et al., 2011) de la siguiente manera:

AO natural azul mensual

= Oferta natural mensual - Caudal Ecológico mensual 
issn 1665-4412, e-issn 2521-9758

Arenas-Jiménez, C. F., Correa-Torres, S. N., \&

Pineda-Vargas, S. M.
Posteriormente, se calcula la Escasez de agua azul mensual (EAAzul), con los datos obtenidos anteriormente:

\section{$E A A z u l=\frac{\Sigma H H \text { azul mensual }}{\text { OA natural y regulada (azul mensual })}$}

Si el EAAzul>1, el uso de agua azul no es sostenible, ya que es mayor el requerimiento de agua que la oferta que puede dar la cuenca. Si el EAAzul<1, significa que los requerimientos no sobrepasan la oferta, de modo que la $\mathrm{HH}$ azul es sostenible.

Sostenibilidad de la HH gris. Se determina mediante el Nivel de contaminación del agua (NCA), el cual se calcula dividiendo la sumatoria de $\mathrm{HH}$ gris mensual, entre la oferta natural regulada $R$ total mensual (embalses y trasvases) disponible para diluir el mayor contaminante (Hoekstra et al., 2011). Para el caso de estudio en la zona alta de la Cuenca de la Quebrada La Angula no hay presencia de estructuras como embalses o trasvases, así que finalmente la oferta natural para comparar con la $\mathrm{HH}$ gris fue la diferencia entre la oferta de agua azul menos la azul, pues este último volumen ya se ha consumido:

$$
N C A \text { mensual }=\frac{\sum H H \text { gris mensual }}{R \text { total mensual }}
$$

\section{RESULTADOS}

Se estimó el régimen de caudales para la zona alta de la Cuenca de La Quebrada La Angula para caudales seco, medio y húmedo de acuerdo con el método de aproximación hidrológica "detallada" en Salinas-Rodríguez (2011). A partir de los caudales medios mensuales se determinó un porcentaje de $15 \%$ correspondiente al caudal ecológico para un estado de conservación moderado de la cuenca según Salinas-Rodríguez (2011) de acuerdo con la metodología de aproximación hidrológica "de gran visión" (figura 1).

Para la zona alta de la Quebrada La Angula se estimó que para la producción de lima Tahití durante un año húmedo (2007) la $\mathrm{HH}$ total fue de $1,737,144,968 \mathrm{~m}^{3}$, teniendo un área cultivada de 240 ha, una producción de 3.840 t y un rendimiento de 16 t/ha. Para un año seco (2015), la HH total fue de $12,499,284,209 \mathrm{~m}^{3}$ para un área de $1179.5 \mathrm{ha}$, una producción de 25713.1 † y un rendimiento de 21.8 †/ ha. La distribución de las azul, verde y gris para cada uno de los años analizados (figura 2A y 2B), donde se aprecia cómo la verde, directamente relacionada con la precipitación efectiva de la zona, posee un porcentaje mucho más grande cuando el régimen de precipitaciones anuales es alto (figura 2A). Cuando las lluvias son escasas, se hace necesario el uso directo de agua de alguna fuente como embalses, ríos o lagunas, por lo cual el porcentaje de $\mathrm{HH}$ azul aumenta considerablemente. En el caso de la zona de estudio, para el año seco se incrementó el consumo de la captación directa de la Quebrada La Angula (figura 2B).

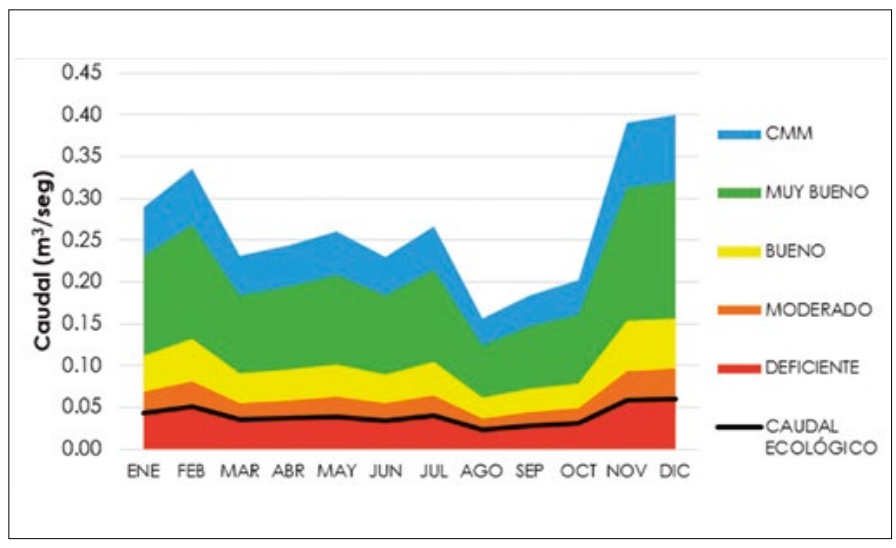

Figura 1. Caudal ecológico conforme objetivos ambientales y sus valores de referencia para la zona alta de la Cuenca de la Quebrada La Angula, Santander, Colombia (CMM: caudal medio mensual).

Elaboración propia.

\section{Análisis de sostenibilidad}

Para el año húmedo (2007), comparada con el año seco (2015), en la zona de estudio no estaba tan intensificada la actividad agrícola. Adicionalmente, para el primer año se registró un valor de precipitación anual de $1030.6 \mathrm{~mm}$, mientras que para el segundo fueron $675.1 \mathrm{~mm}$. En las figuras 3,4 y 5 se presenta la comparación entre cada una de las $\mathrm{HH}$ (verde, azul y gris) respecto a la oferta de agua disponible para cada caso durante el año húmedo (2007). Tanto en la azul, como en la verde y gris, el análisis de sostenibilidad resulta positivo; es decir, en ninguno de los meses analizados del año el consumo sobrepasa la oferta disponible. 
Arenas-Jiménez, C. F., Correa-Torres, S. N., \& Pineda-Vargas, S. M.

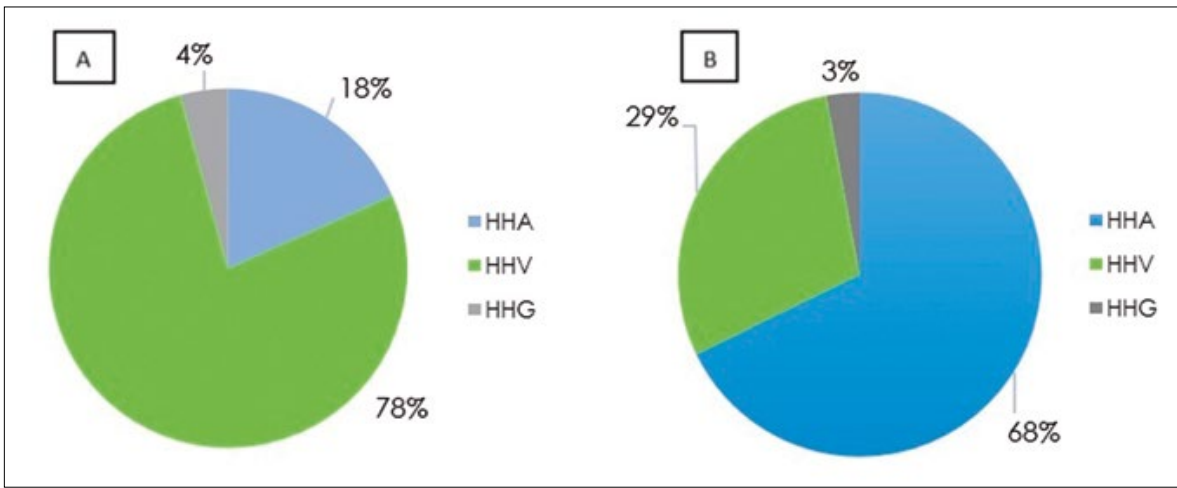

Figura 2. Distribución de la huella hídrica en la zona alta de la Cuenca la Angula, Santander, Colombia, según tipo de huella y tipo de cultivo. A) Para el año 2007 (húmedo) y B) para el año 2015 (seco). Elaboración propia.

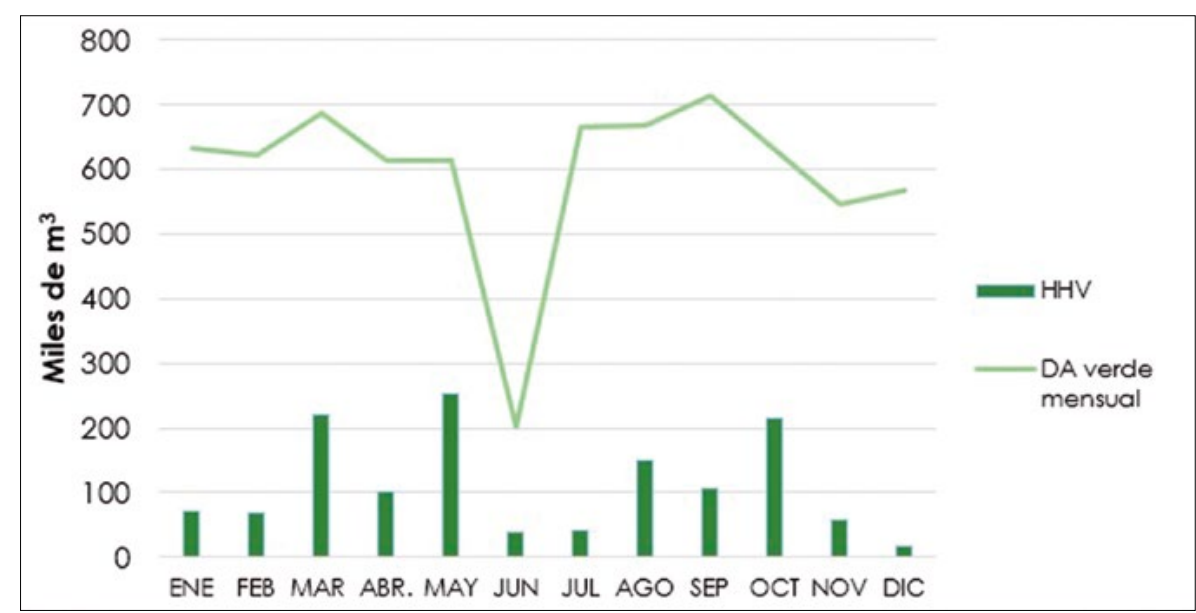

Figura 3. Huella hídrica verde versus disponibilidad de agua verde mensual (DAV) de la zona alta de la Cuenca de la Quebrada La Angula durante el año húmedo (2007). Elaboración propia.

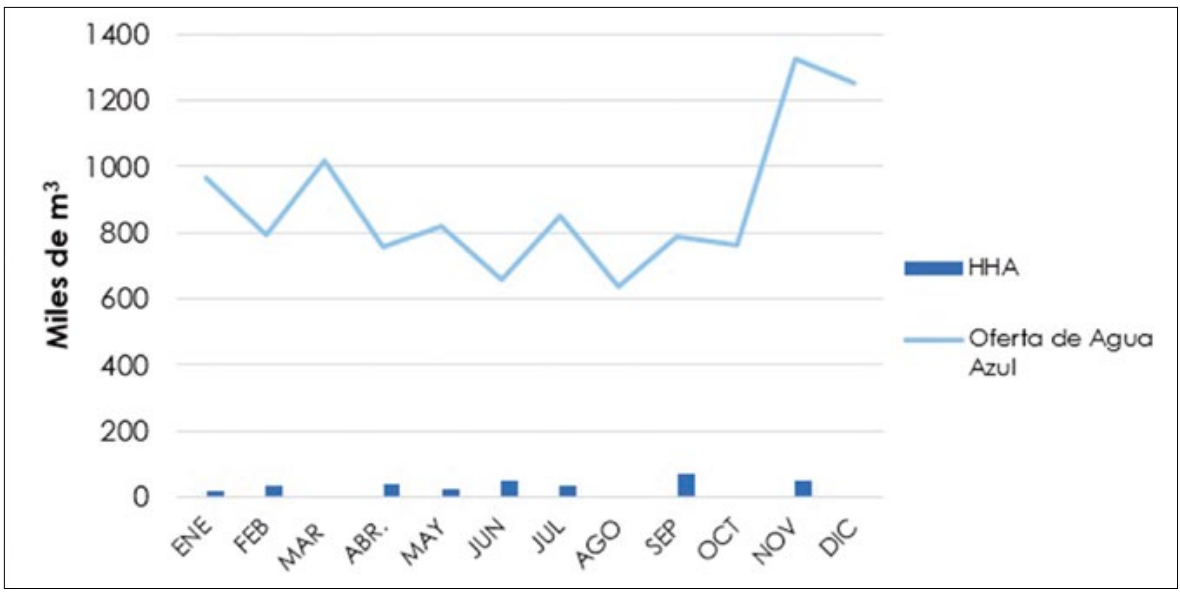

Figura 4. Huella hídrica azul versus oferta de agua azul neta de la zona alta de la Cuenca de la Quebrada La Angula durante el año húmedo (2007).

Elaboración propia. 


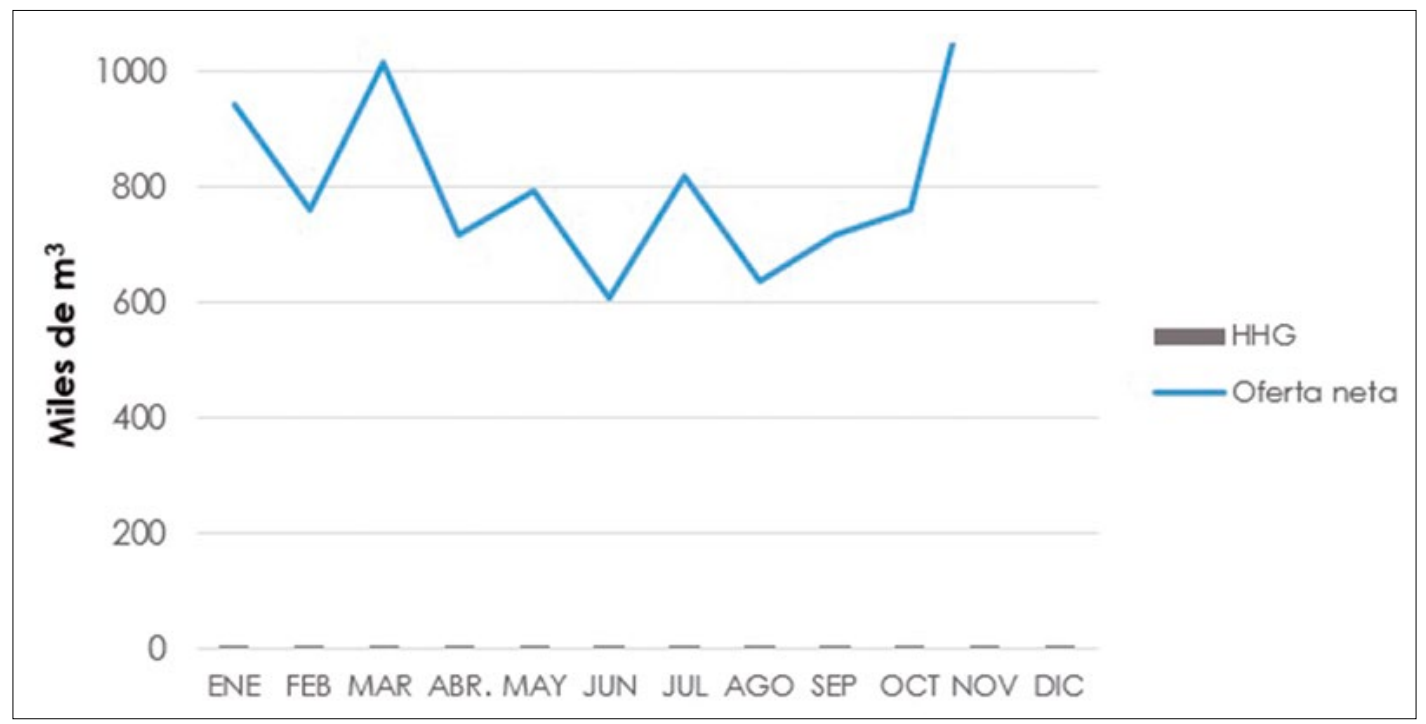

Figura 5. Huella hídrica gris versus oferta natural de la zona alta de la Cuenca de la Quebrada La Angula durante el año húmedo (2007).

Elaboración propia.

Para el caso del año seco (2015) se presentó el fenómeno El Niño (año de sequía en Colombia), que afectó principalmente el segundo semestre. Asimismo, el aumento en la extensión cultivada ocasionó repercusiones negativas en el consumo sostenible del agua. En las figuras 6 y 7 se muestra el análisis de sostenibilidad para la verde y azul durante el año seco. En el caso de la verde, esta nunca supera la disponibilidad de agua, de modo que la sostenibili-

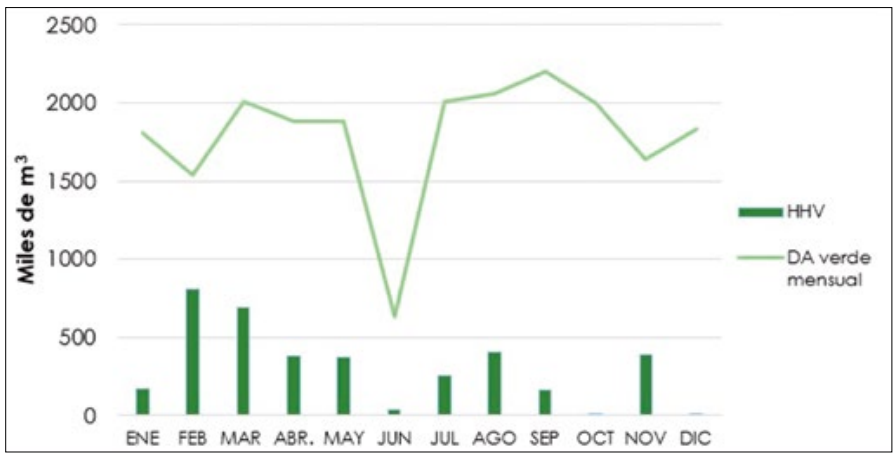

Figura 6. Huella hídrica verde versus disponibilidad de agua verde mensual (DAV) de la zona alta de la Cuenca de la Quebrada La Angula durante el año seco (2015).

Elaboración propia. dad es positiva. Por su parte, la azul supera la oferta de agua de la Quebrada La Angula la mayor parte del año, generando puntos críticos en casi todos los meses. Para el caso de 2015 la afectación llegó a su estado más grave en el mes de octubre, donde prácticamente la oferta de agua azul era nula. Sin embargo, el consumo de agua para cultivos se dio pues los cultivadores tomaron el agua proveniente de las fuentes que drenan a la Quebrada La Angula.

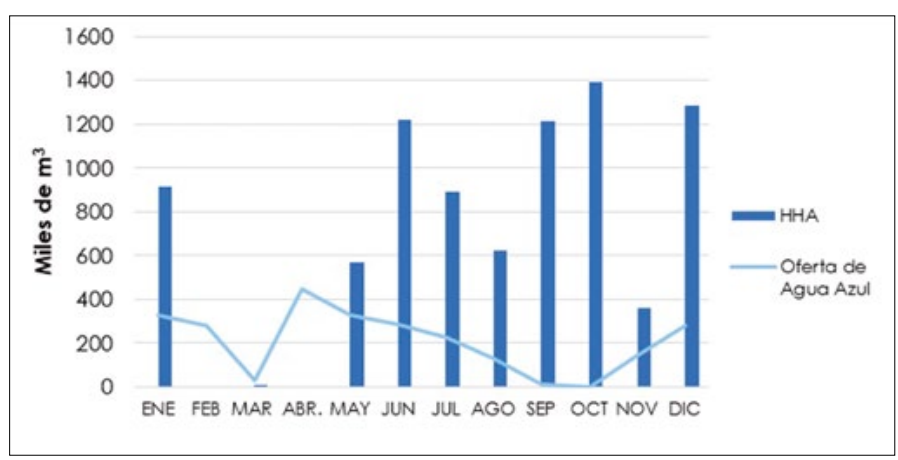

Figura 7. Huella hídrica azul versus oferta de agua azul neta de la zona alta de la Cuenca de la Quebrada La Angula durante el año seco (2015).

Elaboración propia. 
DISCUSIÓN

Los valores de $\mathrm{HH}$ por tonelada para la lima Tahití en la zona de estudio durante los años húmedo y seco fueron comparados con los valores reportados por Mekonnen y Hoekstra (2011) según la base de datos de la FAOSTAT. Estos datos representan valores promedios globales y se registran con los valores calculados en el presente estudio (tabla 2).

Respecto al cultivo de lima Tahití, las diferencias más importantes se presentan en la verde por tonelada para el año seco y en la azul para el año húmedo. Esta última representó cerca de la mitad del valor global, mientras que la huella del seco es bastante cercana, correspondiendo a $93.52 \%$ del promedio global. El contenido de agua gris es inferior al valor promedio global, reportado por Mekonnen y Hoekstra (2011). Esto podría deberse a que en el presente estudio solo se tuvo en cuenta la lixiviación del nitrógeno según Builes Cedula (2013).

Según resultados de Bolaños (2011) y en la comparación con los valores reportados por Mekonnen y Hoekstra (2010) en relación con diferentes tipos de cultivo, los valores en general para países latinoamericanos son más bajos que el promedio global estimado según la FAOSTAT. Esto indica que los datos de huella virtual para el cultivo de lima Tahití en la zona alta de la Cuenca La Angula sí pueden ser inferiores respecto al promedio global y que podrían llegar a ser más bajos en condiciones de altas precipitaciones.

Según el Estudio Nacional del Agua (ENA) en su versión de 2014 (IDEAM, 2015) los cítricos tuvieron una huella hídrica nacional de cerca de $770 \mathrm{Hm}^{3}$ para el 2012. Aunque el consumo que representan los cítricos es superado ampliamente por cultivos como el café, la palma de aceite, el plátano y la caña, los cítricos están entre los 10 cultivos permanentes con más consumo de agua en el territorio colombiano (IDEAM, 2015). En la edición de 2018 los cítricos han aumentado su consumo a cerca $890 \mathrm{Hm}^{3}$, estando todavía entre los 10 cultivos permanentes con mayor huella hídrica en el país (IDEAM, 2019).

Santander es uno de los cuatro departamentos que poseen mayores demandas de agua en el país con $7.8 \%$, a la vez que de los que presenta más susceptibilidad al desabastecimiento (IDEAM, 2019), por lo cual requiere especial atención en el modo en que se está desarrollando la gestión del recurso hídrico. En el caso de la zona de estudio, los cultivos de lima Tahití representan cerca de $25 \%$ del total del área cultivada, lo que implica que uno de los consumos más importantes de agua en la delimitación sea precisamente en las actividades relacionadas con la producción de dicho cítrico.

Tabla 2

Comparación de huella hídrica por tonelada respecto al promedio mundial

\begin{tabular}{|c|c|c|c|}
\hline & $\begin{array}{l}\text { Promedio } \\
\text { global* }^{*}\end{array}$ & $\begin{array}{l}\text { Zona alta Cuenca La } \\
\text { Angula } 2007\end{array}$ & $\begin{array}{c}\text { Zona alta Cuenca La } \\
\text { Angula } 2015\end{array}$ \\
\hline & $\left(m^{3} / t\right)$ & $\left(m^{3} / t\right)$ & $\left(m^{3} / t\right)$ \\
\hline $\mathrm{HH}$ Verde & 432 & 350 & 329.67 \\
\hline $\mathrm{HH}$ AzUl & 152 & 82.88 & 142.15 \\
\hline HH Gris & 58 & 19.51 & 14.32 \\
\hline HH Total & 642 & 452.39 & 486.14 \\
\hline
\end{tabular}

Nota: Adaptada de Mekonnen y Hoekstra (2011). 
issn 1665-4412, e-issn 2521-9758

Arenas-Jiménez, C. F., Correa-Torres, S. N., \&

Pineda-Vargas, S. M.

60

Los resultados del presente artículo muestran el cambio temporal de la $\mathrm{HH}$ en la zona alta de la Cuenca La Angula, comprobando que existen tres variables fundamentales que determinan la $\mathrm{HH}$ agrícola. En primer lugar está el tipo de cultivo, pues dependiendo de la especie se tiene un consumo y una evaporación diferente (FAO, 2006). Asimismo, al comparar los resultados de 2007 con los de 2015, el aumento en el área cultivada como segunda variable, implica un mayor consumo de recurso hídrico. Por último la precipitación, pues en temporadas con mayor régimen de lluvias los cultivos consumen más $y$, por tanto, aumenta la $\mathrm{HH}$ verde (Hoekstra et al., 2011).

Resulta importante ir actualizando los cálculos de $\mathrm{HH}$ para la zona y para los diferentes cultivos que existen desde 2015 hasta ahora, de modo que la misma pueda funcionar como una herramienta de gestión del recurso hídrico. Lo más importante de la $\mathrm{HH}$ no es el consumo en sí mismo, sino el análisis de sostenibilidad, pues al encontrar puntos críticos es posible encontrar medidas que prevengan o mitigan los impactos generados a la cuenca, no solo desde el punto de vista ambiental, sino social y económico; de modo que se pueda, por ejemplo, regular las extensiones y tipos de cultivo para no afectar su sostenibilidad a la vez que se fomente la protección

y preservación de los ecosistemas naturales, ya que en el caso de la zona alta de la Cuenca La Angula (figura 8) las extensiones de zonas explotadas por actividades agrícolas aumentan a la par del crecimiento demográfico de los municipios cercanos. La HH representa un indicador básico para que la academia y las autoridades ambientales trabajen en conjunto con las comunidades agrícolas.

\section{CONCLUSIONES}

Para 2007 la HH total en la zona alta de la Cuenca La Angula para el cultivo de lima Tahití fue de $1.74 \times 10^{6}$ $\mathrm{m}^{3}$, correspondiente a $18 \%$ azul, $78 \%$ verde y $4 \%$ gris. La huella virtual para dicho año fue de $452.4 \mathrm{~m}^{3} / \mathrm{t}$. Para el año 2015, la $\mathrm{HH}$ total para el mismo cultivo fue de $1.25 \times 10^{7} \mathrm{~m}^{3}$ con una distribución de $68 \%$ azul, $29 \%$ verde y $3 \%$ gris. La huella virtual para este año fue de $486.2 \mathrm{~m}^{3} / \mathrm{t}$. El año húmedo (2007) presentó indicadores positivos de sostenibilidad, mientras que el seco (2015) presentó hotspots en el análisis de $\mathrm{HH}$ azul, al punto de afectarse totalmente la oferta de agua azul. El cálculo de la HH representa un indicador de gran utilidad para la elaboración de medidas de gestión para reducir los impactos negativos y mejorar la sostenibilidad en una cuenca.

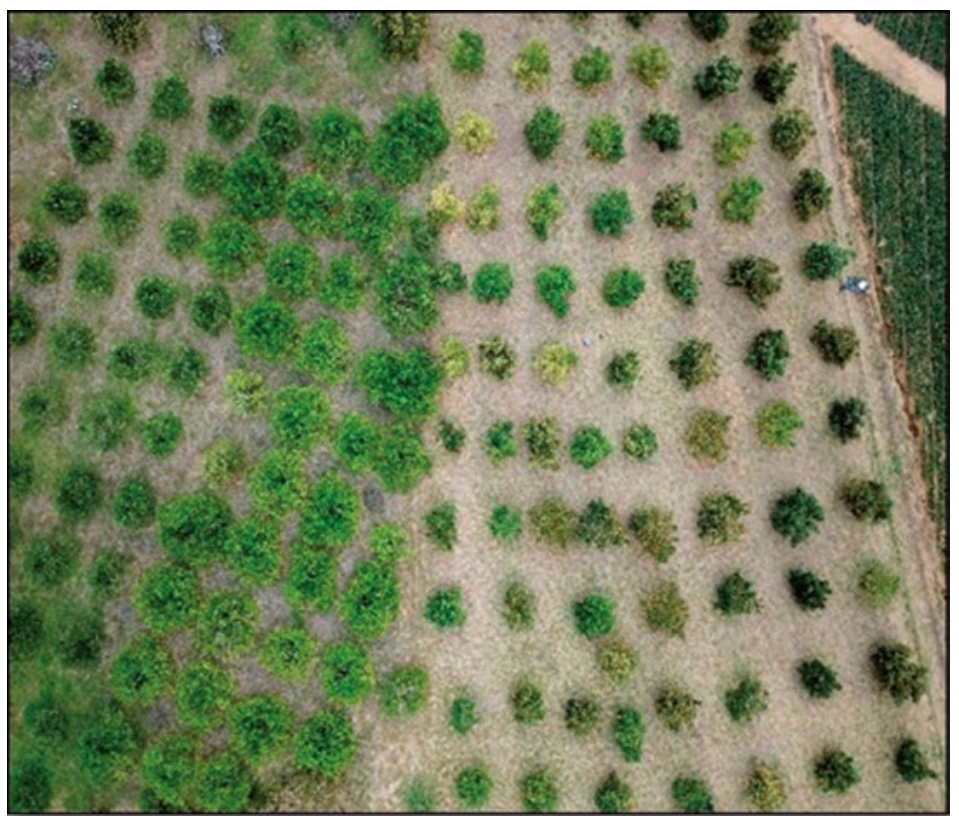

Figura 8. Cultivos de lima Tahití en la zona alta de la Cuenca de la Quebrada La Angula.

Fotografía del equipo de investigación. 
- Arévalo Uribe, D., \& Sabogal Mogollón, J. (2012). Una mirada a la agricultura de Colombia desde su huella hídrica. Colombia: WWF.

- Bolaños, M. E. (2011). Determinación de la huella hídrica y comercio de agua virtual de los principales productos de Honduras. Honduras: Universidad Zamorano.

- Builes Cedula, E. D. (2013). Cuantificación y análisis de sostenibilidad ambiental de la huella hídrica agrícola y pecuaria de la cuenca del río Porce (Tesis de maestría). Recuperada de Repositorio digital de la Universidad Nacional de Colombia bdigital.

- Campuzano, C., Ochoa, J., Guzmán, A., \& Rodríguez, C. (2015). Evaluación multisectorial de la huella hídrica en Colombia: Resultados por subzonas hidrográficas en el marco del Estudio Nacional del Agua 2014. Medellín, Colombia: Instituto de Hidrología, Meteorología y Estudios Ambientales.

- Corporación Autónoma Regional para la Defensa de la Meseta de Bucaramanga. (2004). Plan de ordenamiento y manejo ambiental Subcuenca Lebrija Alto [Documento en pdf]. Bucaramanga, Colombia: CDMB.

- Corporación Colombiana de Investigación Agropecuaria-Corporación Autónoma Regional para la Defensa de la Meseta de Bucaramanga. (1997). Estudio semidetallado de suelos de la Subcuenca de la Quebrada La Angula (123 pp.). Bucaramanga, Colombia: CORPOICA-CDMB.

- DANE. (2015). Cultivo del limón o lima Tahití (Citrus latifolia Tanaka) frente a los efectos de las condiciones climáticas adversas. Boletín mensual. Insumos y Factores Asociados a la Producción Agropecuaria, 41.

- Hoekstra, A. Y. (Ed.). (2003). Virtual water trade. Proceedings of the International Expert Meeting on Virtual Water Trade. En Value of Water Research (Report Series No. 12). Delft, Netherlands: IHE Delft. (2017). Water Footprint Assessment: Evolvement of a New Research Field. Water Resources Management, 31(10), 3061-3081. doi: 10.1007/s11269-017-1618-5
- Hoekstra, A. Y., \& Chapagain, A. K. (2006). Water footprints of nations: Water use by people as a function on their consumption pattern. Water Resources Management, 21, 35-48. doi: 10.1007/s11269-006-9039-x

- Hoekstra, A. Y., Chapagain, A. K., Aldaya, M. M., \& Mekonnen, M. M. (2011). The water footprint assessment manual. London-Washington D.C.: Earthscan.

- Instituto de Hidrología, Meteorología y Estudios Ambientales. (2015). Estudio Nacional del Agua 2014. Bogotá D.C., Colombia: IDEAM. D.C., Colombia: IDEAM.

(2019). Estudio Nacional del Agua 2018. Bogotá

- Mekonnen, M. M., \& Hoekstra, A. Y. (2010). The green, blue and grey water footprint of crops and derived crops products. En Value of Water Research (Report Series No. 47). Delft, Netherlands: UNESCO-IHE.

(2011). The green, blue and grey water footprint of crops and derived crop products. Hidrology and Earth System Sciences, 15, 1577-1600. doi: 10.5194/hess-15-15772011

- Organización de las Naciones Unidas para la Alimentación y la Agricultura. (2006). Evapotranspiración del cultivo. Guía para la determinación de los requerimientos de agua de los cultivos. Estudio FAO riego y drenaje, 56.

- Salinas-Rodríguez, S. (2011). Guía rápida para la determinación de caudales ecológicos. México: Alianza WWF-Fundación Gonzalo Ríos Arronte, I.A.P.

- Velázquez, E. (2008). Agua virtual, huella hídrica y el binomio agua-energía: Repesando los conceptos (12 pp.). Sevilla, España: Universidad Pablo de Olavide. Recuperado de http://hispagua.cedex.es/sites/default/files/hispagua_documento/agua_virtual.pdf

- Zhang, Y., Huang, K., Yu, J., \& Yang, B. (2017). Mapping of water footprint research: A bibliometric analysis during 2006-2015. Journal of Cleaner Production, 149, 70-79. doi: 10.1016/j.jclepro.2017.02.067 